ANNALES

POLONICI MATHEMATICI

XLVIII (1988)

\title{
Bounded solutions of systems of differential equations with impulses
}

\author{
by S. G. Hristova and D. D. Bainov (Plovdiv)
}

\begin{abstract}
Bounded solutions of systems of differential equations with impulses are investigated. A special class of partially continuous functions of Lyapunov type has been introduced by means of which some sufficient as well as necessary and sufficient conditions for the existence of bounded solutions of the system considered have been obtained.
\end{abstract}

1. Introduction. Systems with impulses describe evolutional processes which in certain moments of their evolution rapidly change their state. By the mathematical modelling of such processes it is convenient to neglect the duration of this rapid change and to assume that the process changes its state by jumps.

Mathematical theory of systems of differential equations with impulses marks the beginning of its development by the works of Milman and Myshkis [3], [4]. In spite of the great possibilities of application, this theory develops with comparatively slow paces. This is due to the great difficulties of theoretical and technical nature caused by phenomena such as "beating" of the solutions, merging of the solutions, bifurcations, loss of the property of being autonomous, etc.

In the present paper by means of partially continuous functions of Lyapunov type bounded solutions of differential equations with impulses are investigated. We shall note that such an approach to the investigation of solutions of ordinary differential equations has been used by Yoshizawa [6]-[8].

The use of continuous functions of Lyapunov by the investigation of the solutions of differential equations with impulses whose solutions are partially continuous functions considerably decreases the possibilities of application of the direct method of Lyapunov (cf. [1]).

2. Basic definitions and notations. Consider the system with impulses

$$
\begin{aligned}
& \dot{x}=f(t, x) \quad \text { for } t \neq t_{i}(x), \\
& \left.\Delta x\right|_{t=t_{i}(x)}=I_{i}(x),
\end{aligned}
$$


where $x \in \boldsymbol{R}^{n}, f:[0, \infty) \times \boldsymbol{R}^{n} \rightarrow \boldsymbol{R}^{n}, I_{\mathrm{i}}: \boldsymbol{R}^{n} \rightarrow \boldsymbol{R}^{n}(i=1,2, \ldots), t_{\mathrm{i}}: \boldsymbol{R}^{n} \rightarrow[0, \infty)$, $\left.\Delta x\right|_{r=e}=x(\varrho+0)-x(\varrho-0)$.

Define the sets

$$
\begin{aligned}
& \sigma_{i}=\left\{(t, x) \in[0, \infty) \times R^{n}: t=t_{i}(x)\right\}, \\
& G_{i}=\left\{(t, x) \in[0, \infty) \times R^{n}: t_{i-1}(x)<t<t_{i}(x)\right\}, \\
& D_{i}=\left\{(t, x) \in[0, \infty) \times R^{n}: t_{i-1}(x)<t \leqslant t_{i}(x)\right\}, \quad i \geqslant 1 .
\end{aligned}
$$

We shall say that conditions (A) are satisfied if the following conditions hold:

A1. The functions $t_{i}$ are continuous and

$$
\begin{gathered}
0<t_{1}(x)<t_{2}(x)<\ldots<t_{k}(x)<\ldots \quad \text { for } x \in R^{n}, \\
\inf \left\{t_{j}(x)-t_{j-1}(x): j \geqslant 2, x \in R^{n}\right\}>0, \quad t_{0}(x) \equiv 0 .
\end{gathered}
$$

A2. Uniformly on $x \in R^{n}$ exists the limit $\lim _{i \rightarrow \infty} t_{i}(x)=\infty$.

A3. The integral curves of system (1) meet each hypersurface $\sigma_{1}$ at most once.

We shall note that sufficient conditions for the fulfilment of condition A3 have been given in [2].

Let $x \in R^{n}$. By $\|x\|$ we shall note any norm in $\boldsymbol{R}^{n}$.

Introduce the following sets:

$C_{0}(x)$ is the set of all continuous functions $f:[0, \infty) \times R^{n} \rightarrow R^{n}$ which are locally Lipschitz on their second argument;

$M_{0}$ is the set of all continuous functions $I_{i}: R^{n} \rightarrow R^{n}$;

$K_{0}$ is the set of all monotonely increasing continuous functions $\varphi:[0, \infty) \rightarrow[0, \infty)$,

$$
\begin{gathered}
B_{\alpha}=\left\{x \in R^{n}:\|x\| \leqslant \alpha\right\}, \\
S_{a}=\left\{(t, x):(t, x) \in[0, \infty) \times B_{\alpha} \text { for }(t, x) \in \bigcup_{i=1}^{\infty} G_{i},\right. \\
\left.\left(t, x+I_{k}(x)\right) \in[0, \infty) \times B_{\alpha} \text { for }(t, x) \in \sigma_{k}\right\} .
\end{gathered}
$$

The solution of system (1) with initial condition $x\left(t_{0}\right)=x_{0}$ will be denoted by $x\left(t, \cdot t_{0}, x_{0}\right)$ and the maximal interval on the right of $t_{0}$ in which this solution exists - by $J\left(t_{0}, x_{0}\right) \subset\left[t_{0}, \infty\right)$.

We shall give the definitions of the basic types of boundedness of the solutions of the system with impulses (1) which are analogues to the definitions introduced in [6] for the solutions of ordinary differential equations. 
Definition 1. The solution $x\left(t ; t_{0}, x_{0}\right)$ of system $(1)$ is called bounded if $\left(\exists \beta\left(t_{0}, x_{0}\right)>0\right)\left(\forall t \geqslant t_{0}\right)\left\|x\left(t ; t_{0}, x_{0}\right)\right\|<\beta\left(t_{0}, x_{0}\right)$.

Definition 2. The solutions of system (1) are called equi-bounded if

$$
\begin{aligned}
(\forall \alpha>0)\left(\forall t_{0} \geqslant 0\right)\left(\exists \beta\left(t_{0}, \alpha\right)>0\right)\left(\forall x_{0}:\left(t_{0}, x_{0}\right) \in S_{\alpha}\right)\left(\forall t \geqslant t_{0}\right) & \\
& \left\|x\left(t ; t_{0}, x_{0}\right)\right\|<\beta\left(t_{0}, \alpha\right) .
\end{aligned}
$$

Definition 3. The solutions of system (1) are called uniform-bounded if

$$
(\forall \alpha>0)(\exists \beta(\alpha)>0)\left(\forall\left(t_{0}, x_{0}\right) \in S_{a}\right)\left(\forall t \geqslant t_{0}\right)\left\|x\left(t ; t_{0}, x_{0}\right)\right\|<\beta(\alpha) .
$$

DEFINITION 4. The solutions of system (1) are called ultimately bounded if

$$
\begin{array}{r}
(\exists B>0)\left(\forall\left(t_{0}, x_{0}\right) \in[0, \infty) \times R^{n}\right)\left(\exists T\left(t_{0}, x_{0}\right)>0\right)\left(\forall t \geqslant t_{0}+T\left(t_{0}, x_{0}\right)\right) \\
\left\|x\left(t ; t_{0}, x_{0}\right)\right\|<B .
\end{array}
$$

Definition 5. The solutions of systems (1) are called equiultimately bounded for bound $B$ if

$$
\begin{array}{r}
(\forall \alpha>0)\left(\forall t_{0} \geqslant 0\right)\left(\exists T\left(t_{0}, \alpha\right)>0\right)\left(\forall x_{0}:\left(t_{0}, x_{0}\right) \in S_{\alpha}\right)\left(\forall t \geqslant t_{0}+T\left(t_{0}, \alpha\right)\right) \\
\left\|x\left(t, \cdot t_{0}, x_{0}\right)\right\|<B .
\end{array}
$$

DEFINITION 6. The solutions of system (1) are called uniform-ultimately bounded for bound $B$ if

$$
\begin{array}{r}
(\forall \alpha>0)\left(\forall t_{0} \geqslant 0\right)(\exists T(\alpha)>0)\left(\forall\left(t_{0}, x_{0}\right) \in S_{\alpha}\right)\left(\forall t \geqslant t_{0}+T(\alpha)\right) \\
\left\|x\left(t ; t_{0}, x_{0}\right)\right\|<B .
\end{array}
$$

We shall say that the function $V: \Omega \rightarrow[0, \infty), \Omega \subset[0, \infty) \times \boldsymbol{R}^{n}$ belongs to the class $W$ if the following conditions hold:

1. The function $V(t, x)$ is continuous for $(t, x) \in G_{i} \cap \Omega, i=1,2, \ldots$

2. For $(\varrho, y) \in \sigma_{i} \cap \Omega, i \geqslant 1$ the limits

$$
\lim _{\substack{(t, x) \rightarrow\left((,, y) \\(t, x) \in G_{i}\right.}} V(t, x)=V(\varrho-0, y), \quad \lim _{\substack{(t, x) \rightarrow(e, y) \\(t, x) \in G_{i}+1}} V(t, x)=V(\varrho+0, y)
$$

exist and are finite and the following equality holds:

$$
V(\varrho-0, y)=V(\varrho, y) .
$$

3. The function $V(t, x)$ is locally Lipschitz on its second argument for $(t, x) \in \bigcup_{i=1}^{\infty} G_{i} \cap \Omega$.

Further on we shall use the following notations:

$$
\begin{aligned}
\dot{V}_{(1)}(t, x) & =\varlimsup_{h \rightarrow 0+} \frac{1}{h}\{V(t+h, x+h f(t, x))-V(t, x)\}, \\
V^{\prime}\left(t, x\left(t ; t_{0}, x_{0}\right)\right) & =\varlimsup_{h \rightarrow 0+} \frac{1}{h}\left\{V\left(t+h, x\left(t+h ; t_{0}, x_{0}\right)\right)-V\left(t, x\left(t ; t_{0}, x_{0}\right)\right)\right\} .
\end{aligned}
$$


From condition 3 it follows that if $V \in W$, then the equality $\dot{V}_{(1)}(t, x)$ $=V^{\prime}\left(t, x\left(t ; t_{0}, x_{0}\right)\right)$ holds for $x=x\left(t ; t_{0}, x_{0}\right)$.

If the function $V \in W$ satisfies the conditions

$$
\dot{V}_{(1)}(t, x) \leqslant 0 \quad \text { for }(t, x) \in \bigcup_{i=1}^{\infty} G_{i} \cap \Omega
$$

and

$$
V\left(t+0, x+I_{i}(x)\right) \leqslant V(t, x) \quad \text { for }(t, x) \in \sigma_{i}, i=1,2, \ldots,
$$

and $x\left(t ; t_{0}, x_{0}\right)$ is a solution of system (1), then

$$
V\left(t_{1}, x\left(t_{1} ; t_{0}, x_{0}\right)\right) \leqslant V\left(t_{2}, x\left(t_{2} ; t_{0}, x_{0}\right)\right) \text { for } t_{0} \leqslant t_{2} \leqslant t_{1} .
$$

We shall note that partially continuous functions of Lyapunov type have been used in [5] for the investigation of stability of the solutions of differential equations with impulses.

3. Main results. We shall find out some relations between the various types of boundedness of the solutions of system (1).

LEMMA 1. Let the following conditions be satisfied:

1. Conditions (A) hold.

2. The functions $I_{i}: \boldsymbol{R}^{n} \rightarrow \boldsymbol{R}^{n}(i=1,2, \ldots)$ are of the class $M_{0}$.

3. The function $f:[0, \infty) \times \boldsymbol{R}^{n} \rightarrow \boldsymbol{R}^{n}$ is of the class $C_{0}(x)$.

4. The solution $x\left(t ; t_{0}, x_{0}\right)$ of system (1) is defined for $t \in\left[t_{0}, T\right]$, where $\left(t_{0}, x_{0}\right) \in[0, \infty) \times \boldsymbol{R}^{n}$ is a fixed point, $T=$ const $>0$.

Then for each number $\alpha>0$ there exists a number $\beta\left(t_{0}, \alpha\right)>0$ such that for $\left(t_{0}, x_{0}\right) \in S_{\alpha}$ the inequality $\left\|x\left(t ; t_{0}, x_{0}\right)\right\|<\beta$ holds for $t \in\left[t_{0}, T\right]$.

Proof. Assume that the solution $x\left(t ; t_{0}, x_{0}\right)$ of system (1) for $t \in\left[t_{0}, T\right]$ meets the hypersurfaces $\sigma_{j_{i}}, i=\overline{1, p}$ respectively in the points $\varrho_{1}<\varrho_{2}<\ldots$ $<\varrho_{p}$. By condition A2 we have $p<\infty$. From the continuity of the solution $x\left(t ; t_{0}, x_{0}\right)$ in the interval $\left[t_{0}, \varrho_{1}\right]$ it follows that there exists a constant $\beta\left(t_{0}, \alpha\right)>0$ such that $\left\|x\left(t ; t_{0}, x_{0}\right)\right\|<\beta$ for $t \in\left[t_{0}, \varrho_{1}\right]$. From condition 2 of Lemma 1 it follows that there exists a constant $K_{1}(\beta)>0$ such that for $\|x\|<\beta$ the inequality $\left\|I_{j_{1}}(x)\right\| \leqslant K_{1}$ holds. Hence $\left\|x\left(\varrho_{1}+0\right)\right\|=$ $\left\|x\left(\varrho_{1}\right)+I_{j_{1}}\left(\varrho_{1}\right)\right\| \leqslant \beta+K_{1}$ and there exists a constant $\bar{\beta}\left(t_{0}, \alpha\right)$ such that

$\left\|x\left(t ; t_{0}, x_{0}\right)\right\|=\left\|x\left(t ; \varrho_{1}+0, x\left(\varrho_{1}+0 ; t_{0}, x_{0}\right)\right)\right\| \leqslant \bar{\beta} \quad$ for $t \in\left(\varrho_{1}, \varrho_{2}\right]$.

Applying the above arguments consecutively to each interval $\left(\varrho_{i}, \varrho_{i+1}\right], i=\overline{1, p-1}$, we obtain the assertion of Lemma 1 .

THEOREM 1. Let the following conditions be satisfied:

1. Conditions 1, 2 and 3 of Lemma 1 hold.

2. The solutions of system (1) are equiultimately bounded for bound B. 
Then the following assertions are true:

$(\alpha)$ The solutions of system (1) are equi-bounded.

( $\beta$ ) For any two numbers $\alpha>0$ and $\sigma>0$ there exists a number $T(\sigma, \alpha)>0$ such that for $\left(t_{0}, x_{0}\right) \in S_{a}, t \geqslant t_{0}+T(\sigma, \alpha)$ the inequality $\left\|x\left(t ; t_{0}, x_{0}\right)\right\|<B$ holds.

Proof. Proof of assertion $(\alpha)$. Let $\alpha>0$ be a fixed number. Choose a point $\left(t_{0}, x_{0}\right) \in S_{x}$. By condition 2 of Theorem 1 there exists a number $T\left(t_{0}, \alpha\right)>0$ such that for $t \geqslant t_{0}+T$ the inequality $\left\|x\left(t ; t_{0}, x_{0}\right)\right\|<B$ holds. From Lemma 1 it follows that there exists a number $\beta\left(t_{0}, \alpha\right)>0$ such that $\left\|x\left(t ; t_{0}, x_{0}\right)\right\|<\beta\left(t_{0}, \alpha\right)$ for $t \in\left[t_{0}, t_{0}+T\right]$. Introduce the notation $\gamma\left(t_{0}, \alpha\right)$ $=\max \left(B, \beta\left(t_{0}, \alpha\right)\right)$. Then for $t \geqslant t_{0}$ the inequality $\left\|x\left(t ; t_{0}, x_{0}\right)\right\|<\gamma\left(t_{0}, \alpha\right)$ is satisfied.

Proof of assertion ( $\beta$ ). Let $\alpha>0$ and $\sigma>0$ be fixed numbers and $t_{0} \in[0, \sigma]$. From condition 3 of Theorem 1 it follows that there exists a number $T_{1}(\sigma, \alpha)>0$ such that

$$
\left\|x\left(t ; t_{0}, x_{0}\right)\right\|=\left\|x\left(t ; \sigma, x\left(\sigma ; t_{0}, \alpha\right)\right)\right\|<B
$$

for $t \geqslant \sigma+T_{1}$, where $\left(t_{0}, x_{0}\right) \in S_{\alpha}$. Introduce the notation $T(\sigma, \alpha)=\sigma+$ $+T_{1}(\sigma, \alpha)$. Then for $t \geqslant t_{0}+T(\sigma, \alpha),\left(t_{0}, x_{0}\right) \in S_{\alpha}$ the inequality $\left\|x\left(t ; t_{0}, x_{0}\right)\right\|$ $<B$ is satisfied.

This completes the proof of Theorem 1.

We shall investigate the boundedness of the solutions of the system with impulses (1) by means of functions of the class $W$.

In the further considerations we shall use the following lemma.

LEMMA 2. Let the following conditions be fulfilled:

1. Conditions $\mathrm{A} 1$ and $\mathrm{A} 2$ hold.

2. The function $V:[0, \infty) \times \boldsymbol{R}^{n} \rightarrow[0, \infty)$ is of the class $W$.

Then for any number $\alpha>0$ and for $t_{0} \in[0, \infty)$ there exists a number $K\left(t_{0}, \alpha\right)>0$ such that for $\|x\| \leqslant \alpha$ the following inequality is satisfied

$$
V\left(t_{0}, x\right) \leqslant K\left(t_{0}, \alpha\right) .
$$

Proof. Suppose that the claim is not true, i.e., that there exists a number $\alpha>0$ and points $x_{n} \in R^{n}$ such that $x_{n} \neq x_{k}$ for $n \neq k,\left\|x_{n}\right\| \leqslant \alpha(n$ $=1,2, \ldots)$ and

$$
V\left(t_{0}, x_{n}\right) \geqslant n, \quad n=1,2, \ldots
$$

The sequence $\left\{x_{n}\right\}_{1}^{\infty}$ is bounded, hence there exists a convergent subsequence whose indices we again denote by $n$. Introduce the notation $\beta$ $=\lim _{n \rightarrow \infty} x_{n}$. 
Case 1. Assume that there exists a positive integer $k$ such that $\left(t_{0}, \beta\right) \in G_{k}$, i.e., $t_{k-1}(\beta)<t_{0}<t_{k}(\beta)$. The continuity of the functions $t_{k-1}(x)$ and $t_{k}(x)$ implies that

$$
\lim _{n \rightarrow \infty} t_{k-1}\left(x_{n}\right)=t_{k-1}(\beta) \quad \text { and } \quad \lim _{n \rightarrow \infty} t_{k}\left(x_{n}\right)=t_{k}(\beta) .
$$

From the last two equalities and the inclusion $\left(t_{0}, \beta\right) \in G_{k}$ it follows that for sufficiently large values of $n$ the inclusion $\left(t_{0}, x_{n}\right) \in G_{k}$ holds. Then the following equality $\lim _{n \rightarrow \infty} V\left(t_{0}, x_{n}\right)=V\left(t_{0}, \beta\right)$ is satisfied which contradicts inequality (2).

Case 2. Assume that there exists a positive integer $k$ such that $\left(t_{0}, \beta\right) \in \sigma_{k}$, i.e., $t_{k}(\beta)=t_{0}$. The continuity of the functions $t_{j}(x)(j \geqslant 1)$ implies the relations

$$
\begin{aligned}
\lim _{n \rightarrow \infty} t_{k}\left(x_{n}\right) & =t_{k}(\beta)=t_{0}, \\
\lim _{n \rightarrow \infty} t_{k+1}\left(x_{n}\right) & =t_{k+1}(\beta)>t_{k}(\beta)=t_{0}, \\
\lim _{n \rightarrow \infty} t_{k-1}\left(x_{n}\right) & =t_{k-1}(\beta)<t_{0} .
\end{aligned}
$$

Hence there exist infinitely many members $\bar{x}_{n}$ of the sequence $\left\{x_{n}\right\}_{1}^{\infty}$ satisfying one of the following two assertions:

1. $\bar{x}_{n} \in G_{k}$ for $n=1,2, \ldots$

or

2. $\bar{x}_{n} \in G_{k+1}$ for $n=1,2, \ldots$

Then we have

$$
\lim _{n \rightarrow \infty} V\left(t_{0}, \bar{x}_{n}\right)= \begin{cases}V\left(t_{0}-0, \beta\right) & \text { for } \bar{x}_{n} \in G_{k}, \quad n=1,2, \ldots \\ V\left(t_{0}+0, \beta\right) & \text { for } \bar{x}_{n} \in G_{k+1}, n=1,2, \ldots\end{cases}
$$

Inequality (2) and equality (3) contradict the condition that the limits $V\left(t_{0}-0, \beta\right)$ and $V\left(t_{0}+0, \beta\right)$ are finite.

Lemma 2 is proved.

LEMMA 3. Let the following conditions be satisfied:

1. Conditions (A) hold.

2. The function $f:[0, \infty) \times R^{n} \rightarrow R^{n}$ is of the class $C_{0}(x)$.

3. The functions $I_{i}: \boldsymbol{R}^{n} \rightarrow \boldsymbol{R}^{n}(i=1,2, \ldots)$ are of the class $M_{0}$.

4. The solution $x\left(t ; t_{0}, x_{0}\right)$ of system (1) is strictly bounded by a constant $\beta$ for $t \in J\left(t_{0}, x_{0}\right)$, where $\left(t_{0}, x_{0}\right) \in[0, \infty) \times R^{n}$.

Then $J\left(t_{0}, x_{0}\right)=\left[t_{0}, \infty\right)$, i.e., the solution $x\left(t ; t_{0}, x_{0}\right)$ is defined for $t \geqslant t_{0}$.

Proof. Assume that there exists a number $a>0$ such that $J\left(t_{0}, x_{0}\right)$ $=\left[t_{0}, a\right)$. From the fact that $\lim _{i \rightarrow \infty} t_{i}(x)=\infty$ uniformly on $x \in \boldsymbol{R}^{n}$ it follows that 
for $t \in\left[t_{0}, a\right)$ the solution $x\left(t ; t_{0}, x_{0}\right)$ meets a finite number of hypersurfaces $\sigma_{n_{1}}, \sigma_{n_{2}}, \ldots, \sigma_{n_{p}}$ respectively in the points $\varrho_{1}<\varrho_{2}<\ldots<\varrho_{p}, \varrho_{i} \in\left(t_{0}, a\right)$. Consider the solution $\bar{x}(t)$ of the system without impulses $\dot{x}=f(t, x)$ with initial condition $x\left(\varrho_{p}\right)=x\left(\varrho_{p} ; t_{0}, x_{0}\right)+I_{n_{p}}\left(x\left(\varrho_{p} ; t_{0}, x_{0}\right)\right)$. In virtue of condition 4 the solution $x\left(t ; t_{0}, x_{0}\right)$ is defined for $t \in\left[\varrho_{p}, a\right]$, i.e., $\bar{x}(a)<\infty$. The fact that $x\left(t ; t_{0}, x_{0}\right)=\bar{x}(t)$ for $t \in\left(\varrho_{p}, a\right)$ implies that we can put $x\left(a ; t_{0}, x_{0}\right)$ $=\bar{x}(a)<\infty$, i.e., $J\left(t_{0}, x_{0}\right)=\left[t_{0}, a\right]$ which contradicts the assumption that $J\left(t_{0}, x_{0}\right)=\left[t_{0}, a\right)$.

This completes the proof of Lemma 3.

THEOREM 2. Let the following conditions be satisfied:

1. Conditions (A) hold.

2. The functions $I_{i}: \boldsymbol{R}^{n} \rightarrow \boldsymbol{R}^{n}(i=1,2, \ldots)$ are of the class $M_{0}$.

3. The function $f:[0, \infty) \times \boldsymbol{R}^{n} \rightarrow \boldsymbol{R}^{n}$ is of the class $C_{0}(x)$.

4. There exists a function $V:[0, \infty) \times \boldsymbol{R}^{n} \rightarrow[0, \infty), V \in W$ with the properties:

(i) $a(\|x\|) \leqslant V(t, x)$ for $(t, x) \in[0, \infty) \times R^{n}$, where $a \in K$ and $a(r) \rightarrow \infty$ for $r \rightarrow \infty$ :

(ii) $\dot{V}_{(1)}(t, x) \leqslant 0$ for $(t, x) \in \bigcup_{i=1}^{\infty} G_{i}$;

(iii) $V\left(t+0, x+I_{j}(x)\right) \leqslant V(t, x)$ for $(t, x) \in \sigma_{j}, j \geqslant 1$.

Then the solutions of system (1) are equi-bounded.

Proof. Let $\alpha>0$ be an arbitrary number and $\left(t_{0}, x_{0}\right) \in S_{\alpha}$.

Case 1. Assume that $\left(t_{0}, x_{0}\right) \in \bigcup_{i=1}^{\infty} G_{i}$.

In virtue of Lemma 2 there exists a constant $K\left(t_{0}, \alpha\right)>0$ such that $V\left(t_{0}, x_{0}\right) \leqslant K\left(t_{0}, \alpha\right)$. Choose a number $\beta>0$ such that $a(\beta)>K\left(t_{0}, \alpha\right)$.

Assume that there exists a number $\varrho \in J\left(t_{0}, x_{0}\right)$ such that $\left\|x\left(\varrho ; t_{0}, x_{0}\right)\right\|$ $\geqslant \beta$. Then the following inequalities hold

$$
\begin{aligned}
a(\beta) & \leqslant a\left(\left\|x\left(\varrho ; t_{0}, x_{0}\right)\right\|\right) \leqslant V\left(\varrho, x\left(\varrho ; t_{0}, x_{0}\right)\right) \\
& \leqslant V\left(t_{0}, x_{0}\right) \leqslant K\left(t_{0}, \alpha\right) .
\end{aligned}
$$

The contradiction obtained shows that the assumption is not true.

Case 2. Assume that $\left(t_{0}, x_{0}\right) \in \sigma_{k}$. Then $\left\|x_{0}+I_{k}\left(x_{0}\right)\right\| \leqslant \alpha$ and $V\left(t_{0}+0\right.$, $\left.x_{0}+I_{k}\left(x_{0}\right)\right) \leqslant K\left(t_{0}, \alpha\right)$. In a manner analogous to that in case 1 we get to a contradiction.

Hence $\left\|x\left(t ; t_{0}, x_{0}\right)\right\|<\beta$ for $t \in J\left(t_{0}, x_{0}\right)$. In virtue of Lemma 3 the equality $J\left(t_{0}, x_{0}\right)=\left[t_{0}, \infty\right)$ is fulfilled.

Theorem 2 is proved. 
THEOREM 3. Let the following conditions be satisfied:

1. Conditions (A) hold.

2. The function $f:[0, \infty) \times \boldsymbol{R}^{n} \rightarrow \boldsymbol{R}^{n}$ is of the class $C_{0}(x)$.

3. The functions $I_{i}: \boldsymbol{R}^{\boldsymbol{n}} \rightarrow \boldsymbol{R}^{n}(i=1,2, \ldots)$ are of the class $M_{0}$ and for $\|x\| \leqslant H$ the inequality $\left\|x+I_{i}(x)\right\|<H \quad(i=1,2, \ldots)$ holds, where $H=$ const $>0$.

4. There exists a function $V:[0, \infty) \times\left\{x \in \boldsymbol{R}^{n}:\|x\| \geqslant H\right\} \rightarrow[0, \infty)$ with the properties $V \in W$;

(i) $a(\|x\|) \leqslant V(t, x) \leqslant b(\|x\|)$ for $(t, x) \in \Omega=[0, \infty) \times\{x:\|(x)\| \geqslant H\}$, where $a, b \in K$ and $a(r) \rightarrow \infty$ for $r \rightarrow \infty$;

(ii) $\dot{V}_{(1)}(t, x) \leqslant 0$ for $(t, x) \in \bigcup_{i=1}^{\infty} G_{i} \cap \Omega$;

(iii) $V\left(t+0, x+I_{j}(x)\right) \leqslant V(t, x)$ for $(t, x) \in \sigma_{j} \cap \Omega, j \geqslant 1$.

Then the solutions of system (1) are uniform-bounded.

Proof. Let $t_{0} \geqslant 0,\left(t_{0}, x_{0}\right) \in S_{\alpha}$, where $\alpha>0$ is an arbitrary number. If for $t \in J\left(t_{0}, x_{0}\right)$ the inequality $\left\|x\left(t ; t_{0}, x_{0}\right)\right\|<H$ is fulfilled, then the assertion of the theorem is true. Assume that $H \leqslant\left\|x_{0}\right\|<\alpha$.

Choose a number $\beta(\alpha)$ such that $b(\alpha)<a(\beta)$. Assume that there exists a number $\tau \in J\left(t_{0}, x_{0}\right)$ such that $\left\|x\left(\tau ; t_{0}, x_{0}\right)\right\| \geqslant \beta$. Condition 3 implies the existence of points $\varrho, \eta \in J\left(t_{0}, x_{0}\right)$ such that the inequalities

$$
\begin{aligned}
H \leqslant\left\|x\left(\varrho ; t_{0}, x_{0}\right)\right\| \leqslant \alpha, \quad\left\|x\left(\eta ; t_{0}, x_{0}\right)\right\| \geqslant \beta, \\
\left(\eta, x\left(\eta ; t_{0}, x_{0}\right)\right) \in \bigcup_{i=1}^{\infty} G_{i} \quad \text { and } \quad\left\|x\left(t ; t_{0}, x_{0}\right)\right\| \geqslant H
\end{aligned}
$$

hold for $t \in[\varrho, \eta]$. Condition 4 of Theorem 3 implies the inequality

$$
a(\beta) \leqslant V\left(\eta, x\left(\eta ; t_{0}, x_{0}\right)\right) \leqslant V\left(\varrho, x\left(\varrho ; t_{0}, x_{0}\right)\right) \leqslant b(\alpha)
$$

which contradicts the choice of the number $\beta$.

Hence for $t \in J\left(t_{0}, x_{0}\right)$ the inequality $\left\|x\left(t ; t_{0}, x_{0}\right)\right\|<\beta$ holds. Then Lemma 3 implies the equality $J\left(t_{c}, x_{0}\right)=\left[t_{0}, \infty\right)$.

This completes the proof of Theorem 3.

THEOREM 4. Let the following conditions be satisfied:

1. Conditions 1, 2 and 3 of Theorem 3 hold.

2. There exists a function $V:[0, \infty) \times\{x:\|x\| \geqslant H\} \rightarrow[0, \infty)$ with the properties $V \in W$,

(i) $a(\|x\|) \leqslant V(t, x) \leqslant b(\|x\|)$ for $(t, x) \in \Omega=[0, \infty) \times\{x:\|x\| \geqslant H\}$, where $a, b \in K$ and $a(r) \rightarrow \infty$ for $r \rightarrow \infty$;

(ii) $\dot{V}_{(1)}(t, x) \leqslant-c(\|x\|)$ for $(t, x) \in \bigcup_{i=1}^{\infty} G_{i} \cap \Omega$, where $c(r)>0$ is a continuous function;

(iii) $V\left(t+0, x+I_{j}(x)\right) \leqslant V(t, x)$ for $(t, x) \in \sigma_{j} \cap \Omega, j \geqslant 1$.

Then the solutions of system (1) are uniform-ultimately bounded. 
Proof. Let $\alpha>0$ be an arbitrary number and $\left(t_{0}, x_{0}\right) \in S_{\alpha}$. In virtue of Theorem 3 the solutions of system (1) are uniform-bounded, i.e., there exists a number $\beta(\alpha)>0$ such that $\left\|x\left(t ; t_{0}, x_{0}\right)\right\|<\beta$ for $t \geqslant t_{0}$. Moreover, $\beta>\alpha$ $\geqslant H$. From the uniform boundedness of the solutions of system (1) it follows that there exists a number $B>0$ such that for $\left(t_{0}, y\right) \in S_{H}$ and $t \geqslant t_{0}$ the inequality $\left\|x\left(t ; t_{0}, y\right)\right\|<B$ is satisfied. Assume that for $t \geqslant t_{0}$ the inequality $\left\|x\left(t ; t_{0}, x_{0}\right)\right\|>H$ holds. From properties (ii) and (iii) of the function $V(t, x)$ it follows that there exists a number $\gamma(\alpha)>0$ such that for $H \leqslant\|x\| \leqslant \beta$ the inequality $V(t, x) \leqslant V\left(t_{0}, x_{0}\right)-\gamma(\alpha)\left(t-t_{0}\right), t \geqslant t_{0}$ is satistied. From last inequality, property (i) and the assumption that $\left\|x\left(t ; t_{0}, x_{0}\right)\right\|>H$ follows the inequality

$$
\begin{aligned}
a(H) & \leqslant V\left(t, x\left(t ; t_{0}, x_{0}\right)\right) \leqslant V\left(t_{0}, x_{0}\right)-\gamma(\alpha)\left(t-t_{0}\right) \\
& \leqslant b(\alpha)-\gamma(\alpha)\left(t-t_{0}\right) .
\end{aligned}
$$

If in inequality (4) we choose $t>t_{0}+T(\alpha)$, where

$$
T(\alpha)=\frac{b(\alpha)-a(H)}{\gamma(\alpha)},
$$

this leads to a contradiction. Hence there exists a number $\varrho$ such that $\left\|x\left(\varrho ; t_{0}, x_{0}\right)\right\|<H$.

If $\left(t_{0}+T, x\left(t_{0}+T ; t_{0}, x_{0}\right)\right) \in \bigcup_{i=1}^{\infty} G_{i}$, then there exists a number $\tau \in$ $\left[t_{0}, t_{0}+T\right)$ such that for $t \geqslant \tau$ the inequality $\left\|x\left(t ; t_{0}, x_{0}\right)\right\|<B$ is satisfied.

If $\left(t_{0}+T, x\left(t_{0}+T ; t_{0}, x_{0}\right)\right) \in \sigma_{k}$, then there exists a sufficiently small number $\varepsilon>0$ such that $\left\|x\left(t_{0}+T_{1} ; t_{0}, x_{0}\right)\right\| \leqslant H$ where $T_{1}(\alpha)=T(\alpha)+\varepsilon$. Then for $t \geqslant t_{0}+T_{1}(\alpha)$ the following inequality holds:

$$
\left\|x\left(t ; t_{0}, x_{0}\right)\right\|<B .
$$

This shows that the solutions of system (1) are uniform-ultimately bounded.

Theorem 4 is proved.

By means of functions of the class $W$ we shall obtain some necessary and sufficient conditions for ultimate boundedness of the solutions of system (1).

THEOREM 5. Let the following conditions be fulfilled:

1. Conditions (A) hold.

2. The function $f:[0, \infty) \times \boldsymbol{R}^{n} \rightarrow \boldsymbol{R}^{n}$ is of the class $C_{0}(x)$.

3. The functions $I_{i}: \boldsymbol{R}^{n} \rightarrow \boldsymbol{R}^{n}(i=1,2, \ldots)$ are of the class $M_{0}$.

Then necessary and sufficient condition for the solutions of system (1) to be equi-ultimately bounded for a bound is the existence of a function $V:[0, \infty) \times \boldsymbol{R}^{n} \rightarrow[0, \infty), V \in W$ with the properties: 
(i) $a(\|x\|) \leqslant V(t, x)$ for $(t, x) \in[0, \infty) \times\{x:\|x\| \geqslant B\}$, where $a \in K$ for $r$ $\geqslant B$ and $a(r) \rightarrow \infty$ for $r \rightarrow \infty$;

(ii) $\dot{V}_{(1)}(t, x) \leqslant-c V(t, x)$ for $(t, x) \in \bigcup_{i=1}^{\infty} G_{i}$, where $c=$ const $>0$;

(iii) $V\left(t+0, x+I_{j}(x)\right) \leqslant V(t, x)$ for $(t, x) \in \sigma_{j}, j \geqslant 1$.

Proof. Sufficiency. Choose a number $\alpha>0$ and a point $\left(t_{0}, x_{0}\right) \in S_{a}$. By Lemma 2 there exists a number $K\left(t_{0}, \alpha\right)>0$ such that $V\left(t_{0}, x_{0}\right) \leqslant K\left(t_{0}, \alpha\right)$. We choose a number

$$
T=T\left(t_{0}, \alpha\right)>\frac{1}{c} \ln \frac{K\left(t_{0}, \alpha\right)}{a(B)} .
$$

From properties (ii) and (iii) of the function $V(t, x)$ it follows that for $t \in J\left(t_{0}, x_{0}\right)$ the following inequality holds:

$$
V\left(t, x\left(t ; t_{0}, x_{0}\right)\right) \leqslant V\left(t_{0}, x_{0}\right) e^{-c\left(t-t_{0}\right)}<a(B) .
$$

From property (i) of the function $V(t, x)$ and the above inequality it follows that

$$
a\left(\left\|x\left(t ; t_{0}, x_{0}\right)\right\|\right) \leqslant V\left(t, x\left(t ; t_{0}, x_{0}\right)\right)<a(B) .
$$

Inequality (5) shows that $\left\|x\left(t ; t_{0}, x_{0}\right)\right\|<B$ for $t \geqslant t_{0}+T, t \in J\left(t_{0}, x_{0}\right)$.

Then by Lemma 3 the relation $J\left(t_{0}, x_{0}\right)=\left[t_{0}, \infty\right)$ holds.

Necessity. Assume that the solutions of system (1) are equi-ultimately bounded for bound $B^{\prime}$. Choose a number $\sigma>0$. Introduce the notation $\Omega_{\sigma, \alpha}=S_{\alpha} \cap\left\{[0, \sigma] \times R^{n}\right\}$. By assertion $(\alpha)$ of Theorem 1 the solutions of system (1) are equi-bounded, i.e., there exists a constant $\beta(\sigma, \alpha)$ such that $\left\|x\left(t ; t_{0}, x_{0}\right)\right\|<\beta$ for $t \geqslant t_{0},\left(t_{0}, x_{0}\right) \in \Omega_{\sigma, \alpha}$. From assertion $(\beta)$ of Theorem 1 it follows that there exists a constant $T(\sigma, \alpha)>0$ such that $\left\|x\left(t ; t_{0}, x_{0}\right)\right\|<B^{\prime}$ for $t \geqslant t_{0}+T(\sigma, \alpha),\left(t_{0}, x_{0}\right) \in \Omega_{\sigma, \alpha}$.

Define the function $G:[0, \infty) \rightarrow[0, \infty)$ by the equality

$$
G(u)= \begin{cases}u-B^{\prime} & \text { for } u \geqslant B^{\prime}, \\ 0 & \text { for } 0 \leqslant u<B^{\prime} .\end{cases}
$$

Let $(t, x) \in \Omega_{\sigma, \alpha}$ be an arbitraty point and $(t, x) \in D_{i}$. Define the functions

$$
\begin{aligned}
V_{i}(t, x) & =\sup _{\tau}\left\{G(\|x(t+\tau ; t, x)\|) e^{c \tau}:(t+\tau, x(t+\tau ; t, x)) \in D_{i}\right\}, \\
V_{i+k}(t, x) & =\sup _{\tau \geqslant 0}\left\{G(\|x(t+\tau ; t, x)\|) e^{c \tau}:(t+\tau, x(t+\tau ; t, x)) \in D_{j_{i+k}}\right\}, \\
V(t, x) & =\sup _{k \geqslant i} V_{k}(t, x) .
\end{aligned}
$$

The function $V(t, x)$ satisfies the estimate

$$
V(t, x) \geqslant V_{i}(t, x) \geqslant G(\|x\|) .
$$


Moreover, the function $G(u) \geqslant 0$ for $r \geqslant B$ is a continuous increasing function and $G(u) \rightarrow \infty$ for $u \rightarrow \infty$. Hence the function $V(t, x)$ has property (i).

Let $(t, x) \in \Omega_{\sigma, a},\left(t^{\prime}, x^{\prime}\right) \in \Omega_{\sigma, a}, t<t^{\prime},(t, x),\left(t^{\prime}, x^{\prime}\right) \in G_{i}, t^{\prime}-t<\inf \left\{t_{j+1}(x)-\right.$ $-t_{j}(x): j \geqslant 1, x \in R^{n}$;.

From the condition $\|x(\tau ; t, x)\|<B^{\prime}$ for $\tau \geqslant t+T(\sigma, \alpha)$ it follows that $G(\|x(\tau ; t, x)\|)=0$ for $\tau \geqslant t+T(\sigma, \alpha)$ and

$$
V(t, x)=\max _{i \leqslant k \leqslant i+p} V_{k}(t, x), \quad V\left(t^{\prime}, x^{\prime}\right)=\max _{i \leqslant k \leqslant i+p} V_{k}\left(t^{\prime}, x^{\prime}\right),
$$

where $(t+T, x(t+T ; t, x)) \in D_{j_{i+p+1}}$.

Introduce the notation

$$
F^{*}(\sigma, \alpha)=1+\max _{(t, x) \in[0, \sigma+T] \times B_{\beta}}\|f(t, x)\|
$$

and the set

$$
M_{a}=\{(t, x): t \in[0, \sigma+T(\alpha)],\|x\| \leqslant \beta(\alpha)\} .
$$

The condition $f(t, x) \in C_{0}(x)$ implies the existence of a constant $L(\sigma, \alpha)>0$ such that

$$
\|f(t, x)-f(t, y)\| \leqslant L(\sigma, \alpha)\|x-y\| \quad \text { for }(t, x),(t, y) \in M_{a} .
$$

Let $V(t, x)=V_{k}(t, x)$. If $V_{k}(t, x)=G(\|x(t+\varrho ; t, x)\|) e^{c \varrho}$, where $t+\varrho$ $=t_{k}(x(t+\varrho ; t, x)), t^{\prime}+\varrho=t_{k}\left(x\left(t^{\prime}+\varrho ; t^{\prime}, x^{\prime}\right)\right)$ then the following relations hold

$$
\begin{aligned}
& V(t, x)-V\left(t^{\prime}, x^{\prime}\right) \leqslant V_{k}(t, x)-V_{k}\left(t^{\prime}, x^{\prime}\right) \\
& \leqslant\left\|x(t+\varrho ; t, x)-x\left(t^{\prime}+\varrho ; t^{\prime}, x^{\prime}\right)\right\| e^{c e} \\
& \leqslant e^{c \varrho}\left\{\left\|x(t+\varrho ; t, x)-x\left(t^{\prime}+\varrho ; t, x\right)\right\|+\right. \\
&\left.+\left\|x\left(t^{\prime}+\varrho ; t, x\right)-x\left(t^{\prime}+\varrho ; t^{\prime}, x\right)\right\|+\left\|x\left(t^{\prime}+\varrho ; t^{\prime}, x\right)-x\left(t^{\prime}+\varrho ; t^{\prime}, x^{\prime}\right)\right\|\right\} \\
& \leqslant e^{c T(\sigma, \alpha)}\left\{N(\sigma, \alpha)\left|t-t^{\prime}\right|+e^{L(\sigma, \alpha) T(\sigma, \alpha)}(1+M(\alpha))^{\gamma(\alpha)}\left\|x-x^{\prime}\right\|\right\}
\end{aligned}
$$

where

$$
\begin{gathered}
N(\sigma, \alpha)=F^{*}(\sigma, \alpha)\left\{1+e^{L(\sigma, \alpha) T(\sigma, \alpha)}[1+M(\alpha)]^{\gamma(\alpha)}\right\}, \\
\gamma(\alpha)=\sup _{t \geqslant 0} i(t, t+T(\sigma, \alpha))<\infty, \quad M(\alpha)=\sup _{i} M_{i}(\alpha),
\end{gathered}
$$

$i(t, \varrho)$ is the number of the points $t_{i} \in(t, \varrho)$,

$$
\left\|I_{i}(x)-I_{i}\left(x^{\prime}\right)\right\| \leqslant M_{i}(\alpha)\left\|x-x^{\prime}\right\| \quad \text { for } x, x^{\prime} \in B_{\beta} .
$$

If $V_{k}(t, x)=G(\|x(t+\varrho ; t, x)\|) e^{c \ell}$, where

$$
(t+\varrho ; x(t+\varrho ; t, x)) \in D_{j_{k}}, \quad\left(t^{\prime}+\varrho ; x\left(t^{\prime}+\varrho ; t^{\prime}, x^{\prime}\right)\right) \in D_{j_{k+1}}
$$


then the following relations hold

$$
\begin{aligned}
V(t, x)-V\left(t^{\prime}, x^{\prime}\right) & =V_{k}(t, x)-V_{k+1}\left(t^{\prime}, x^{\prime}\right) . \\
& \leqslant\left\|x(t+\varrho ; t, x)-x\left(t^{\prime}+\varrho ; t^{\prime}, x^{\prime}\right)\right\| e^{c e} \\
& \leqslant e^{c T(\sigma, \alpha)}\left\{N(\sigma, \alpha)\left|t-t^{\prime}\right|+e^{L(\sigma, \alpha) T(\sigma, \alpha)}(1+M(\alpha))^{\gamma(\alpha)}\left\|x-x^{\prime}\right\|\right\} .
\end{aligned}
$$

Analogously we can prove that

$$
V(t, x)-V\left(t^{\prime}, x^{\prime}\right) \geqslant-e^{c T(\sigma, \alpha)}\left\{N(\sigma, \alpha)\left|t-t^{\prime}\right|+e^{L(\sigma, \alpha) T(\sigma, \alpha)}(1+M(\alpha))^{y(\alpha)}\left\|x-x^{\prime}\right\|\right\} .
$$

Hence for $(t, x) \in \bigcup_{i=1}^{\infty} G_{i}$ the function $V(t, x)$ is continuous on both arguments and Lipschitz on its second argument.

We shall show that $V(t, x)$ has property (ii). In fact, for $(t, x) \in \bigcup_{i=1}^{\infty} G_{i}$ and $h>0$ the following inequality holds

$$
\begin{aligned}
V_{k}(t+h, x(t+h ; t, x))= & \sup _{\tau \geqslant 0}\left\{G \left(\|x(t+h+\tau ; t, x)\| e^{c \tau}:\right.\right. \\
& =\sup _{\tau \geqslant h}\left\{G \left(\|x(t+\tau ; t, x)\| e^{\tau \tau} e^{-c h}:\right.\right. \\
& \left.(t+h+\tau, x(t+h+\tau ; t, x)) \in D_{j_{k}}\right\} \\
& \leqslant V_{k}(t, x) e^{-c h} .
\end{aligned}
$$

The above inequality implies that

$$
\varlimsup_{h \rightarrow 0+} \frac{1}{h}\left\{V_{k}(t+h, x(t+h ; t, x))-V_{k}(t, x)\right\} \leqslant-c V_{k}(t, x) .
$$

Hence the function $V(t, x)$ has property (ii).

Finally we shall show that the function $V(t, x)$ has property (iii). In fact, for $(t, x) \in \sigma_{j}$, the following inequality holds

$$
V(t, x)=\sup _{k \geqslant j} V_{k}(t, x) \geqslant \sup _{k \geqslant j+1} V_{k}(t, x)=V\left(t+0, x+I_{j}(x)\right) .
$$

This completes the proof of Theorem 5 .

THEOREM 6. Let the following conditions be fulfilled:

1. Conditions 1, 2 and 3 of Theorem 3 hold.

2. The solutions of system (1) are at the same time uniform-bounded and uniform-ultimately bounded for bound $B$.

Then there exists a function $V:[0, \infty) \times\{x:\|x\| \geqslant H\} \rightarrow[0, \infty), V \in W$, with the properties 
(i) $a\left(\| x\{\mid) \leqslant V(t, x) \leqslant b(\|x\|)\right.$ for $(t, x) \in \Omega=[0, \infty) \times\left\{x:\|x\| \geqslant H_{\}}\right.$, where $a, b \in K$ and $a(r) \rightarrow \infty$ for $r \rightarrow \infty$;

(ii) $\dot{V}_{(1)}(t, x) \leqslant-c V(t, x)$ for $(t, x) \in \bigcup_{i=1}^{\infty} G_{i} \cap \Omega$, where $c=$ const $>0$;

(iii) $V\left(t+0, x+I_{j}(x)\right) \leqslant V(t, x)$ for $(t, x) \in \sigma_{j} \cap \Omega, j \geqslant 1$.

Proof. Let $\delta>1$ be an arbitrary number and the point $(t, x) \epsilon$ $[0, \infty) \times R^{n}$ be such that $\|x\|>\delta B,(t, x) \in D_{i}$. Define the functions

$$
\begin{aligned}
V_{i}(t, x) & =\sup _{\tau}\left\{0\left(\|x(t+\tau ; t, x)\| \frac{1+\delta \tau}{1+\tau}:(t+\tau, x(t+\tau ; t, x)) \in D_{i}\right\},\right. \\
V_{i+k}(t, x) & =\sup _{\tau}\left\{0 x(t+\tau ; t, x) \| \frac{1+\delta \tau}{1+\tau}:(t+\tau, x(t+\tau ; t, x)) \in D_{j_{i+k}}\right\}, \\
V(t, x) & =\sup _{k \geqslant i} V_{k}(t, x),
\end{aligned}
$$

where $H=\delta B$.

The function $V(t, x)$ satisfies the estimate

$$
V(t, x) \geqslant V_{i}(t, x) \geqslant\|x\| .
$$

From the condition that the solutions of system (1) are uniform-bounded it follows that for any number $\alpha>0$ there exists a number $\beta(\alpha)$ such that for $\left(t_{0}, x_{0}\right) \in S_{\alpha}$ and $t \geqslant t_{0}$ the inequality $\left\|x\left(t ; t_{0}, x_{0}\right)\right\|<\beta$ holds. Moreover, we can assume that the function $\beta(\alpha)$ is continuous, monotonely increasing and $\beta(\alpha) \rightarrow \infty$ for $\alpha \rightarrow \infty$. From the definition of the function $V(t, x)$ it follows that

$$
V(t, x) \leqslant \beta(\|x\|) .
$$

Inequalities (6) and (7) show that the function $V(t, x)$ has property (i).

We shall show that the function $V(t, x)$ has property (ii). In fact, let $(t, x) \in G_{i} \cap \Omega$. Then for sufficiently small values of $h>0$ the inclusion $(t+h, x(t+h ; t, x)) \in G_{i} \cap \Omega$ holds and for $k \geqslant i$ the following inequality is satisfied

$$
\begin{aligned}
V_{k}(t+h, x(t+h ; t, x))= & \sup _{\varrho \geqslant h}\left\{\|x(t+\varrho ; t, x)\| \frac{1+\delta \varrho-\delta h}{1+\varrho-h}:\right. \\
& \left.\quad(t+\varrho, x(t+\varrho ; t, x)) \in D_{j_{k}}\right\} \\
& \leqslant(1-\delta h+h) V_{k}(t, x) .
\end{aligned}
$$

From the above inequality it follows that

i.e.,

$$
\frac{1}{h}\left\{V_{k}(t+h, x(t+h ; t, x))-V_{k}(t, x)\right\} \leqslant(1-\delta) V_{k}(t, x),
$$

$$
\dot{V}_{(1)}(t, x) \leqslant(1-\delta) V(t, x)
$$

which shows that the function $V(t, x)$ has property (ii). 
Let $(t, x) \in \sigma_{i} \cap \Omega$. Then the following equalities hold

$$
V(t, x)=\sup _{k \geqslant i} V_{k}(t, x), \quad V\left(t+0, x+I_{i}(x)\right)=\sup _{k \geqslant i} V_{k}(t, x) .
$$

From equalities (8) and the definition of the function $V(t, x)$ we obtain the inequality

$$
V\left(t+0, x+I_{i}(x)\right) \leqslant V(t, x)
$$

which shows that the function $V(t, x)$ has property (iii).

Let $(t, x) \in \Omega_{\sigma, \alpha},\left(t^{\prime}, x^{\prime}\right) \in \Omega_{\sigma, \alpha}, t<t^{\prime},(t, x),\left(t^{\prime}, x^{\prime}\right) \in G_{i}$,

$$
t^{\prime}-t<\inf \left\{t_{j+1}(x)-t_{j}(x): x \in R^{n}, j \geqslant 1\right\},
$$

where $\Omega_{\sigma, \alpha}=\left\{[0, \sigma] \times \boldsymbol{R}^{n}\right\} \times S_{\alpha}$.

From condition 2 of Theorem 6 it follows that there exists a number $T(\alpha)>0$ such that $\|x(\tau ; t, x)\|<B$ for $\tau>t+T(\alpha)$.

Hence for sufficiently large values of $\tau$ the inequality

$$
\|x(\tau ; t, x)\| \frac{1+\delta \tau}{1+\tau} \leqslant \delta B
$$

holds which shows that there exists a positive integer $p$ such that

$$
V(t, x)=\sup _{i \leqslant k \leqslant i+p}(t, x) \quad \text { and } \quad V\left(t^{\prime}, x^{\prime}\right)=\sup _{i \leqslant k \leqslant i+p} V_{k}\left(t^{\prime}, x^{\prime}\right) .
$$

Analogously to the proof of the necessity in Theorem 5 we can prove that the function $V(t, x)$ is continuous on both arguments for $(t, x)$ $\in \bigcup_{i=1}^{\infty} G_{i} \cap \Omega$ and Lipschitz on its second argument.

Theorem 6 is proved.

We shall apply some of the sufficient conditions obtained to the investigation of concrete systems with impulse.

EXample 1. Consider the system with impulses

$$
\begin{aligned}
\dot{x} & =B(t) x+A(t) x \quad \text { for } t \neq t_{i}, \\
\left.\Delta x\right|_{t=t_{j}} & =C_{i} x\left(t_{i}\right),
\end{aligned}
$$

where $x \in R^{n}, B(t)=\operatorname{diag}\left(b_{1}(t), b_{2}(t), \ldots, b_{n}(t)\right), b_{k}(t)<0$ for $t \in[0, \infty), A(t)$ is an $(n \times n)$-matrix which is symmetric, negatively definite and continuous for $t \in[0, \infty), \quad C_{k}=\operatorname{diag}\left(c_{1 k}, \ldots, c_{n k}\right), \quad-1<c_{i k} \leqslant 0, \quad 0<t_{1}<t_{2}<\ldots$, $\lim _{k \rightarrow \infty} t_{k}=\infty$. 

fact,

The function $V(t, x)=\sum_{i=1}^{n} x_{i}^{2}$ satisfies the conditions of Theorem 3. In

$$
\begin{gathered}
\dot{V}_{(9)}(t, x)=2 \sum_{i=1}^{n} b_{i}(t) x_{i}^{2}+2 \sum_{i, j=1}^{n} a_{i j} x_{i} x_{j} \quad \text { for } t \neq t_{j}, \\
V\left(t_{k}+0, x+C_{k} x\right)=\sum_{i=1}^{n}\left[x_{i}\left(C_{i k}+1\right)\right]^{2} \leqslant V\left(b_{k}, x\right), \\
\|x\| \leqslant V(t, x) \leqslant \gamma\|x\|, \quad \gamma>1 .
\end{gathered}
$$

Then by Theorem 3 system (9) has uniform-bounded solutions.

Moreover, if there exist constants $\gamma_{i}>0$ such that $b_{i}(t) \leqslant-\gamma_{i}$, then by Theorem 4 the solutions of system (9) are uniform-ultimately bounded.

In the special case when in system (9) $n=1, B(t)=b, A(t) \equiv 0$, then any of its solutions has the form

$$
x(t)=x_{0} e^{b\left(t-t_{0}\right)} \prod_{t_{0}<t_{i}<t}\left(1+c_{i}\right)
$$

and it is easy to check that these solutions are indeed uniform-bounded.

EXAmple 2. Consider the system with impulses

$$
\begin{gathered}
\dot{x}=a(t) y-b(t) x\left(x^{2}+y^{2}\right), \\
\dot{y}=-a(t) y+b(t) y\left(x^{2}+y^{2}\right) \quad \text { for } t \neq t_{i}, \\
\left.\Delta x\right|_{t=t_{i}}=c_{i} x\left(t_{i}\right),\left.\quad \Delta y\right|_{t=t_{i}}=d_{i} y\left(t_{i}\right),
\end{gathered}
$$

where $a(t), b(t)$ are continuous functions for $t \geqslant 0, b(t) \leqslant 0,-1<c_{k} \leqslant 0$, $-1<d_{k} \leqslant 0, k=1,2, \ldots, 0<t_{1}<t_{2}<\ldots, \lim _{k \rightarrow \infty} t_{k}=\infty$.

The function $V(t, x, y)=x^{2}+y^{2}$ satisfies the conditions of Theorem 3 . In fact,

$$
\begin{gathered}
\dot{V}_{(10)}(t, x, y)=2 b(t)\left(x^{2}+y^{2}\right) \leqslant 0 \quad \text { for } t \neq t_{i}, \\
V\left(t_{i}+0, x+c_{i} x, y+d_{i} y\right)=x^{2}\left(1+c_{i}\right)^{2}+y^{2}\left(1+d_{i}\right)^{2} \leqslant V(t, x, y) .
\end{gathered}
$$

Then by Theorem 3 the solutions of system (10) are uniform-bounded.

Moreover, if there exists a constant $\gamma>0$ such that $b(t) \leqslant-\gamma$, then by Theorem 4 the solutions of system (10) are uniform-ultimately bounded.

\section{References}

[1] O. S. Chernikova, On the dissipativity of systems of differential equations with impulse effect (in Russian), UMJ 5 (1983), 656-660.

[2] A. B. Dishliev and D. D. Bainov, Conditions for the ahsence of the phenomenon "beating" for systems of impulse differential equations, Bull. Inst. Math. Acad. Sinica 13 (1985), $237-256$. 
[3] V. D. Mil'man and A. D. Myshkis, On stability of motions in the presence of impulses (in Russian), Sibirskii Math. J. I (1960), 233-237.

[4] -, -, Random impulses in linear dynamical systems, In: Approximate methods for the solution of differential equations (in Russian), Publ. House Acad. Sci. Ukr. SSR, Kiev 1963, $64-81$.

[5] P. S. Simeonov and D. D. Bainov, Stability with respect to part of the variables in systems with impulse effect, J. Math. Anal. Appl. (1986).

[6] T. Yoshizawa, Liapunov's functions and boundedness of solutions, Funkcial. Ekvac. 2 (1959), 95-142.

[7] -, Stability theory by Liapunov's second method, Math. Soc. Japan (1966).

[8] - Stability theory and the existence of periodic solutions and almost periodic solutions, Springer-Verlag, New-York-Heidelberg-Berlin 1975.

PLOVDIV UNIVERSITY, "PAISSII HILENDARSKI"

Reçu par la Rédaction le 1987.02.09 